\title{
Traveling wave solutions in a higher dimensional lattice competition-cooperation system with stage structure
}

\author{
Yanli He $\mathbb{D}$, Kun $\mathrm{Li}^{*}$ \\ School of Mathematics and Computational Science, Hunan First Normal University, Changsha, Hunan \\ 410205, People's Republic of China
}

\begin{abstract}
In this paper, we consider the existence of traveling wave solutions in a higher dimensional lattice competition-cooperation system with stage structure. We first construct a pair of upper and lower solutions. The upper solutions are allowed to be larger than positive equilibrium point. Then we establish the existence of traveling wave solutions by means of cross iterative and Schauder's fixed point theorem.
\end{abstract}

Mathematics Subject Classification (2010). 37L60, 34K10, 39A10

Keywords. lattice, stage structure, traveling wave solution, Schauder's fixed point theorem, upper and lower solutions

\section{Introduction}

Lattice differential equations are the discrete versions of reaction-diffusion equations. In past few years, many authors have paid their attention on the existence of traveling wave solutions for lattice differential equations, see $[3,4,7,8,13,16,17,21]$ for one or two dimensional lattices and $[15,20,22]$ for higher dimensional lattices, and also see the results for reaction-diffusion equations with or without stage structure $[1,2,5,6,9,10,14,18,19]$.

In this paper we are concerned with the existence of traveling wave solutions of a higher dimensional lattice competition-cooperation system with stage structure

$$
\left\{\begin{array}{l}
u_{1 \eta}^{\prime}(t)=D_{1}\left(\Delta_{n} u_{1}\right)_{\eta}+\alpha_{1} e^{-\gamma_{1} \tau_{1}} u_{1 \eta}\left(t-\tau_{1}\right)-a_{1} u_{1 \eta}^{2}(t)-b_{1} u_{1 \eta}(t) u_{2 \eta}(t) \\
u_{2 \eta}^{\prime}(t)=D_{2}\left(\Delta_{n} u_{2}\right)_{\eta}+\alpha_{2} e^{-\gamma_{2} \tau_{2}} u_{2 \eta}\left(t-\tau_{2}\right)+b_{2} u_{1 \eta}(t) u_{2 \eta}(t)-a_{2} u_{2 \eta}^{2}(t)
\end{array}\right.
$$

where all the parameters are positive constants, $t>0,\left(\Delta_{n} w\right)_{\eta}=\sum_{|\xi-\eta|=1, \xi \in \mathbb{Z}^{n}} w_{\xi}-2 n w_{\eta}$, $\eta \in \mathbb{Z}^{n},|\cdot|$ denotes the Euclidean norm in $\mathbb{R}^{n}, n \in \mathbb{Z}^{+}$. System (1.1) has four equilibria

$$
\mathbf{0}=(0,0), \quad\left(\frac{\alpha_{1} e^{-\gamma_{1} \tau_{1}}}{a_{1}}, 0\right), \quad\left(0, \frac{\alpha_{2} e^{-\gamma_{2} \tau_{2}}}{a_{2}}\right), \quad \mathbf{K}=\left(k_{1}, k_{2}\right),
$$

\footnotetext{
*Corresponding Author.

Email addresses: m15084970596_1@163.com (Y. He), kli@mail.bnu.edu.cn (K. Li)

Received: 02.10.2018; Accepted: 08.08.2019
} 
where

$$
k_{1}=\frac{a_{2} \alpha_{1} e^{-\gamma_{1} \tau_{1}}-b_{1} \alpha_{2} e^{-\gamma_{2} \tau_{2}}}{a_{1} a_{2}+b_{1} b_{2}}>0, \quad k_{2}=\frac{a_{1} \alpha_{2} e^{-\gamma_{2} \tau_{2}}+b_{2} \alpha_{1} e^{-\gamma_{1} \tau_{1}}}{a_{1} a_{2}+b_{1} b_{2}}>0
$$

provided that

$$
a_{2} \alpha_{1} e^{-\gamma_{1} \tau_{1}}>b_{1} \alpha_{2} e^{-\gamma_{2} \tau_{2}}
$$

We are interested in the existence of traveling wave solutions of higher dimensional lattice (1.1) connecting $\mathbf{0}$ with $\mathbf{K}$. In this case, the reaction terms of (1.1) satisfy partial monotonicity. We notice that there are some existence results about traveling wave solutions for systems with partial monotonicity. For example, Huang et al. [8,9] considered the existence of traveling wave solutions of continuous and discrete reaction-diffusion systems with partial monotonicity by using upper and lower solutions and Schauder's fixed point theorem; Li et al. [12] considered the existence and asymptotic behavior of traveling wave solutions of competition-cooperation system on 1D lattice. Recently, Li et al. [11] considered the existence of traveling wave solutions of diffusive and competition-cooperation system with stage structure. However, their results are not applied to system (1.1). Hence we need to extend the above methods to higher dimensional lattice system (1.1).

Motivated by techniques in $[8,9,11,12]$, we will establish the existence of traveling wave solutions of system (1.1) connecting $\mathbf{0}$ with $\mathbf{K}$ by Schauder's fixed point theorem and upper and lower solutions method. The upper solutions are allowed to be larger than positive equilibrium point, which is different from $[8,9]$.

This paper is organized as follows. In Section 2, we first construct a pair of upper and lower solutions and prove the continuity and compactness of operators, then we establish the existence of traveling wave solutions by means of the cross iterative and Schauder's fixed point theorem.

\section{The existence of traveling wave solutions}

In this paper, we use the usual notations for the standard ordering in $\mathbb{R}^{2}$. That is, for $u=\left(u_{1}, u_{2}\right)$ and $v=\left(v_{1}, v_{2}\right)$, we denote $u \leq v$ if $u_{i} \leq v_{i}, i=1,2$, and $u<v$ if $u \leq v$ but $u \neq v$. In particular, we denote $u \ll v$ if $u \leq v$ but $u_{i} \neq v_{i}, i=1,2$. If $u \leq v$, we also denote $(u, v]=\left\{\omega \in \mathbb{R}^{2}, u<\omega \leq v\right\},[u, v)=\left\{\omega \in \mathbb{R}^{2}, u \leq \omega<v\right\}$, $[u, v]=\left\{\omega \in \mathbb{R}^{2}, u \leq \omega \leq v\right\}$. In the following, $|\cdot|$ denotes the Euclidean norm in $\mathbb{R}^{2}$ or $\mathbb{R}^{n}$ and $\|\cdot\|$ denotes the supremum norm in $C\left([-\tau, 0], \mathbb{R}^{2}\right)$.

Let

$$
C_{[\mathbf{0}, \mathbf{M}]}\left(\mathbb{R}, \mathbb{R}^{2}\right)=\left\{(\phi, \psi) \in C\left(\mathbb{R}, \mathbb{R}^{2}\right): \mathbf{0} \leq(\phi(s), \psi(s)) \leq \mathbf{M}, s \in \mathbb{R}\right\},
$$

where $\mathbf{K} \leq \mathbf{M}:=\left(M_{1}, M_{2}\right)$.

Denote

$$
\left\{\begin{array}{l}
f_{1}\left(u_{\eta}(s), v_{\eta}(s)\right)=\alpha_{1} e^{-\gamma_{1} \tau_{1}} u_{\eta}\left(-\tau_{1}\right)-a_{1} u_{\eta}^{2}(0)-b_{1} u_{\eta}(0) v_{\eta}(0), \\
f_{2}\left(u_{\eta}(s), v_{\eta}(s)\right)=\alpha_{2} e^{-\gamma_{2} \tau_{2}} v_{\eta}\left(-\tau_{2}\right)+b_{2} u_{\eta}(0) v_{\eta}(0)-a_{2} v_{\eta}^{2}(0) .
\end{array}\right.
$$

Lemma 2.1. For the functional $f(\phi, \psi)=\left(f_{1}(\phi, \psi), f_{2}(\phi, \psi)\right)$,

(A) there exist $L_{i}>0$ such that

$$
\begin{aligned}
& \qquad\left|f_{i}\left(u_{1 \eta}, v_{1 \eta}\right)-f_{i}\left(u_{2 \eta}, v_{2 \eta}\right)\right| \leq L_{i}\|U-V\| \\
& \text { for } U=\left(u_{1 \eta}, v_{1 \eta}\right), V=\left(u_{2 \eta}, v_{2 \eta}\right) \in C\left([-\tau, 0], \mathbb{R}^{2}\right) \text { with } \\
& \boldsymbol{O} \leq\left(u_{i \eta}(s), v_{i \eta}(s)\right) \leq \boldsymbol{M}, s \in[-\tau, 0], i=1,2,
\end{aligned}
$$

and it satisfies partially quasimonotone condition:

$(\mathrm{PQM})$ there exist $\beta_{1}>0$ and $\beta_{2}>0$ such that

$$
\begin{aligned}
& f_{1}\left(u_{1 \eta}(s), v_{1 \eta}(s)\right)-f_{1}\left(u_{2 \eta}(s), v_{1 \eta}(s)\right)+\beta_{1}\left[u_{1 \eta}(0)-u_{2 \eta}(0)\right] \geq 2 n D_{1}\left[u_{1 \eta}(0)-u_{2 \eta}(0)\right], \\
& f_{1}\left(u_{1 \eta}(s), v_{1 \eta}(s)\right)-f_{1}\left(u_{1 \eta}(s), v_{2 \eta}(s)\right) \leq 0 \\
& f_{2}\left(u_{1 \eta}(s), v_{1 \eta}(s)\right)-f_{2}\left(u_{2 \eta}(s), v_{2 \eta}(s)\right)+\beta_{2}\left[v_{1 \eta}(0)-v_{2 \eta}(0)\right] \geq 2 n D_{2}\left[v_{1 \eta}(0)-v_{2 \eta}(0)\right]
\end{aligned}
$$


for $u_{i \eta}(s), v_{i \eta}(s) \in C([-\tau, 0], \mathbb{R}), i=1,2$, with $\boldsymbol{O} \leq\left(u_{2 \eta}(s), v_{2 \eta}(s)\right) \leq\left(u_{1 \eta}(s), v_{1 \eta}(s)\right) \leq \boldsymbol{M}$ for $s \in[-\tau, 0]$.

Proof. (A) is obvious. Next we check (PQM). For any $\left(u_{1 \eta}, v_{1 \eta}\right),\left(u_{2 \eta}, v_{2 \eta}\right) \in C\left([-\tau, 0], \mathbb{R}^{2}\right)$ with $\mathbf{0} \leq\left(u_{2 \eta}(s), v_{2 \eta}(s)\right) \leq\left(u_{1 \eta}(s), v_{1 \eta}(s)\right) \leq \mathbf{M}$, let $\beta_{1}>0$ and $\beta_{2}>0$ satisfying $\beta_{1}>2 a_{1} M_{1}+b_{1} M_{2}+2 n D_{1}$ and $\beta_{2}>2 a_{2} M_{2}+2 n D_{2}$, we have

$$
\begin{gathered}
f_{1}\left(u_{1 \eta}, v_{1 \eta}\right)-f_{1}\left(u_{2 \eta}, v_{1 \eta}\right)-2 n D_{1}\left[u_{1 \eta}(0)-u_{2 \eta}(0)\right] \\
\geq\left\{-a_{1}\left[u_{1 \eta}(0)+u_{2 \eta}(0)\right]-b_{1} v_{1 \eta}(0)\right\}\left[u_{1 \eta}(0)-u_{2 \eta}(0)\right]-2 n D_{1}\left[u_{1 \eta}(0)-u_{2 \eta}(0)\right] \\
\geq-\left(2 a_{1} M_{1}+b_{1} M_{2}+2 n D_{1}\right)\left[u_{1 \eta}(0)-u_{2 \eta}(0)\right] \\
\geq-\beta_{1}\left[u_{1 \eta}(0)-u_{2 \eta}(0)\right], \\
\quad f_{1}\left(u_{1 \eta}, v_{1 \eta}\right)-f_{1}\left(u_{1 \eta}, v_{2 \eta}\right)=-b_{1} u_{1 \eta}(0)\left[v_{1 \eta}(0)-v_{2 \eta}(0)\right] \leq 0, \\
f_{2}\left(u_{1 \eta}, v_{1 \eta}\right)-f_{2}\left(u_{2 \eta}, v_{2 \eta}\right)-2 n D_{2}\left[u_{1 \eta}(0)-u_{2 \eta}(0)\right] \\
\geq-a_{2}\left[v_{1 \eta}(0)+v_{2 \eta}(0)\right]\left[v_{1 \eta}(0)-v_{2 \eta}(0)\right]-2 n D_{2}\left[v_{1 \eta}(0)-v_{2 \eta}(0)\right] \\
\geq-\left(2 a_{2} M_{2}+2 n D_{2}\right)\left[v_{1 \eta}(0)-v_{2 \eta}(0)\right] \\
\geq-\beta_{2}\left[v_{1 \eta}(0)-v_{2 \eta}(0)\right] .
\end{gathered}
$$

We give the definition of traveling wave solutions of (1.1) .

Definition 2.2. A traveling wave solution of (1.1) has special form $u_{1 \eta}(t)=\phi(\sigma \cdot \eta+$ $c t), u_{2 \eta}(t)=\psi(\sigma \cdot \eta+c t)$, where $\phi( \pm \infty)$ and $\psi( \pm \infty)$ both exist, $\sigma=\left(\sigma_{1}, \sigma_{2}, \cdots, \sigma_{n}\right) \in \mathbb{R}^{n}$ is a unit vector, $c>0$ is the wave speed, $(\phi, \psi)$ is the wave profile.

Denoting $\sigma \cdot \eta+c t$ by $t$, we search for the solutions of system

$$
\left\{\begin{array}{l}
c \phi^{\prime}(t)=D_{1} \sum_{k=1}^{n}\left[\phi\left(t+\sigma_{k}\right)-2 \phi(t)+\phi\left(t-\sigma_{k}\right)\right]+f_{1}^{c}\left(\phi_{t}, \psi_{t}\right), \\
c \psi^{\prime}(t)=D_{2} \sum_{k=1}^{n}\left[\psi\left(t+\sigma_{k}\right)-2 \psi(t)+\psi\left(t-\sigma_{k}\right)\right]+f_{2}^{c}\left(\phi_{t}, \psi_{t}\right)
\end{array}\right.
$$

satisfying

$$
\lim _{t \rightarrow-\infty}(\phi(t), \psi(t))=\mathbf{0}, \quad \lim _{t \rightarrow+\infty}(\phi(t), \psi(t))=\mathbf{K},
$$

where $\phi_{t}(s)=\phi(t+s), \psi_{t}(s)=\psi(t+s), s \in[-\tau, 0], \tau=\max \left\{\tau_{1}, \tau_{2}\right\}, f_{i}^{c}(\phi, \psi), i=1,2$, is defined by

$$
\left\{\begin{array}{l}
f_{1}^{c}\left(\phi_{t}, \psi_{t}\right)=\alpha_{1} e^{-\gamma_{1} \tau_{1}} \phi\left(t-c \tau_{1}\right)-a_{1} \phi^{2}(t)-b_{1} \phi(t) \psi(t), \\
f_{2}^{c}\left(\phi_{t}, \psi_{t}\right)=\alpha_{2} e^{-\gamma_{2} \tau_{2}} \psi\left(t-c \tau_{2}\right)+b_{2} \phi(t) \psi(t)-a_{2} \psi^{2}(t) .
\end{array}\right.
$$

Define $F=\left(F_{1}, F_{2}\right): C_{[\mathbf{0}, \mathbf{M}]}\left(\mathbb{R}, \mathbb{R}^{2}\right) \rightarrow C\left(\mathbb{R}, \mathbb{R}^{2}\right)$ by

$$
F_{i}(\phi, \psi)(t)=\frac{1}{c} e^{-\frac{\beta_{i}}{c} t} \int_{-\infty}^{t} e^{\frac{\beta_{i}}{c} s} H_{i}(\phi, \psi)(s) d s, \quad i=1,2,
$$

where $H=\left(H_{1}, H_{2}\right): C_{[\mathbf{0}, \mathbf{M}]}\left(\mathbb{R}, \mathbb{R}^{2}\right) \rightarrow C\left(\mathbb{R}, \mathbb{R}^{2}\right)$ is defined by

$$
\left\{\begin{array}{l}
H_{1}(\phi, \psi)(t)=f_{1}^{c}\left(\phi_{t}, \psi_{t}\right)+\beta_{1} \phi(t)+D_{1} \sum_{k=1}^{n}\left[\phi\left(t+\sigma_{k}\right)-2 \phi(t)+\phi\left(t-\sigma_{k}\right)\right], \\
H_{2}(\phi, \psi)(t)=f_{2}^{c}\left(\phi_{t}, \psi_{t}\right)+\beta_{2} \psi(t)+D_{2} \sum_{k=1}^{n}\left[\psi\left(t+\sigma_{k}\right)-2 \psi(t)+\psi\left(t-\sigma_{k}\right)\right] .
\end{array}\right.
$$

Then $F$ is well defined and for any $(\phi, \psi) \in C_{[\mathbf{0}, \mathbf{M}]}\left(\mathbb{R}, \mathbb{R}^{2}\right)$, we have

$$
c\left[F_{i}(\phi, \psi)\right]^{\prime}(t)=-\beta_{i} F_{i}(\phi, \psi)(t)+H_{i}(\phi, \psi)(t), \quad i=1,2 .
$$


We only need to find a fixed point of (2.3) satisfying (2.2).

Let $\mu \in\left(0, \min \left\{\beta_{1} / c, \beta_{2} / c\right\}\right)$ and equip $C\left(\mathbb{R}, \mathbb{R}^{2}\right)$ with the norm $|\cdot|_{\mu}$ defined by

$$
|\Phi|_{\mu}=\sup _{t \in \mathbb{R}}|\Phi(t)| e^{-\mu|t|} \quad \text { and } \quad B_{\mu}\left(\mathbb{R}, \mathbb{R}^{2}\right)=\left\{\Phi \in C\left(\mathbb{R}, \mathbb{R}^{2}\right): \sup _{t \in \mathbb{R}}|\Phi(t)| e^{-\mu|t|}<\infty\right\} .
$$

Then it is easy to check that $\left(B_{\mu}\left(\mathbb{R}, \mathbb{R}^{2}\right),|\cdot|_{\mu}\right)$ is a Banach space.

Definition 2.3. A pair of functions $\bar{\Phi}=(\bar{\phi}, \bar{\psi}), \underline{\Phi}=(\phi, \underline{\psi}) \in C\left(\mathbb{R}, \mathbb{R}^{2}\right)$ is called a weak upper solution and a weak lower solution of (2.1), respectively, if there exist finitely many constants $T_{i}, i=1, \cdots, p$ such that $\bar{\Phi}$ and $\underline{\Phi}$ are differentiable in $\mathbb{R} \backslash T, T:=\left\{T_{i}: i=\right.$ $1, \cdots, p\}$ and satisfy

$$
\begin{cases}c \bar{\phi}^{\prime}(t) \geq D_{1} \sum_{k=1}^{n}\left[\bar{\phi}\left(t+\sigma_{k}\right)-2 \bar{\phi}(t)+\bar{\phi}\left(t-\sigma_{k}\right)\right]+f_{1}^{c}\left(\bar{\phi}_{t}, \underline{\psi}_{t}\right) & \text { for } t \in \mathbb{R} \backslash T, \\ c \bar{\psi}^{\prime}(t) \geq D_{2} \sum_{k=1}^{n}\left[\bar{\psi}\left(t+\sigma_{k}\right)-2 \bar{\psi}(t)+\bar{\psi}\left(t-\sigma_{k}\right)\right]+f_{2}^{c}\left(\bar{\phi}_{t}, \bar{\psi}_{t}\right) & \text { for } t \in \mathbb{R} \backslash T,\end{cases}
$$

and

$$
\begin{cases}c \underline{\phi}^{\prime}(t) \leq D_{1} \sum_{k=1}^{n}\left[\underline{\phi}\left(t+\sigma_{k}\right)-2 \underline{\phi}(t)+\underline{\phi}\left(t-\sigma_{k}\right)\right]+f_{1}^{c}\left(\underline{\phi}_{t}, \bar{\psi}_{t}\right) & \text { for } t \in \mathbb{R} \backslash T, \\ c \underline{\psi}^{\prime}(t) \leq D_{2} \sum_{k=1}^{n}\left[\underline{\psi}\left(t+\sigma_{k}\right)-2 \underline{\psi}(t)+\underline{\psi}\left(t-\sigma_{k}\right)\right]+f_{2}^{c}\left(\underline{\phi}_{t}, \underline{\psi}_{t}\right) & \text { for } t \in \mathbb{R} \backslash T .\end{cases}
$$

Next we will construct a pair of upper and lower solutions of (2.1) satisfying

(A1) $\mathbf{0} \leq(\underline{\phi}(t), \underline{\psi}(t)) \leq(\bar{\phi}(t), \bar{\psi}(t)) \leq \mathbf{M}, t \in \mathbb{R}$

(A2) $\lim _{t \rightarrow-\infty}(\bar{\phi}(t), \bar{\psi}(t))=\mathbf{0}, \quad \lim _{t \rightarrow+\infty}(\underline{\phi}(t), \underline{\psi}(t))=\lim _{t \rightarrow+\infty}(\bar{\phi}(t), \bar{\psi}(t))=\mathbf{K}$.

To do this, we need to assume

$$
a_{1} k_{1}>b_{1} k_{2}
$$

which implies (1.2) holds.

Similar to $[11,12]$, it is easy to prove the following lemma.

Lemma 2.4. Let

$$
\Delta_{i}(\lambda, c):=D_{i} \sum_{k=1}^{n}\left(e^{\lambda \sigma_{k}}+e^{-\lambda \sigma_{k}}-2\right)-c \lambda+\alpha_{i} e^{-\gamma_{i} \tau_{i}} e^{-\lambda c \tau_{i}}, \quad i=1,2 .
$$

Then, there exist $c_{1}^{*}>0$ and $c_{2}^{*}>0$ such that $\Delta_{1}(\lambda, c)=0$ and $\Delta_{2}(\lambda, c)=0$, respectively, have only two positive roots $0<\lambda_{1}<\lambda_{3}$ and $0<\lambda_{2}<\lambda_{4}$, and

$$
\Delta_{1}(\lambda, c)\left\{\begin{array} { l } 
{ > 0 , \quad \lambda < \lambda _ { 1 } \text { or } \lambda > \lambda _ { 3 } , } \\
{ < 0 , \quad \lambda _ { 1 } < \lambda < \lambda _ { 3 } , }
\end{array} \quad \text { and } \quad \Delta _ { 2 } ( \lambda , c ) \left\{\begin{array}{ll}
>0, & \lambda<\lambda_{2} \text { or } \lambda>\lambda_{4}, \\
<0, & \lambda_{2}<\lambda<\lambda_{4},
\end{array}\right.\right.
$$

for $0<c<c_{i}^{*}, \Delta_{i}(\lambda, c)=0$ has no real roots on $\mathbb{R}, i=1,2$.

Now we construct a pair of upper and lower solutions for $c>c^{*}:=\max \left\{c_{1}^{*}, c_{2}^{*}\right\}$.

For fixed

$$
\nu \in\left(1, \min \left\{2, \frac{\lambda_{3}}{\lambda_{1}}, \frac{\lambda_{4}}{\lambda_{2}}, \frac{\lambda_{1}+\lambda_{2}}{\lambda_{1}}\right\}\right)
$$

consider $h_{i}(t)=e^{\lambda_{i} t}-q e^{\nu \lambda_{i} t}, \mathrm{i}=1,2$, where $q>1$ is large enough. By direct calculation, it shows that $h_{i}(t)$ has a unique global maximum $\varrho_{i}=\varrho_{i}(q)>0$ at $t_{i}^{*}=t_{i}^{*}(q)=$ $-\frac{1}{(\nu-1) \lambda_{i}} \ln q \nu<0$, and

$$
\lim _{q \rightarrow \infty} \varrho_{i}(q)=\lim _{q \rightarrow \infty} e^{\lambda_{i} t_{i}^{*}(q)}=\lim _{q \rightarrow \infty} q e^{\nu \lambda_{i} t_{i}^{*}(q)}=0, \quad i=1,2 .
$$


We also have $h_{i}(t)$ is increasing in $t \leq t_{i}^{*}$ and decreasing in $t \geq t_{i}^{*}$. So $h_{i}(t)=A_{i}$ has only two different roots $t_{i *}$ and $t_{i}\left(t_{i *}<t_{i}^{*}<t_{i}\right)$ and $t_{i}-t_{i *}>\tilde{t}$, where $A_{i}=e^{\lambda_{i}\left(t_{i}^{*}-\tilde{t}\right)}-$ $q e^{\nu \lambda_{i}\left(t_{i}^{*}-\tilde{t}\right)}>0, \tilde{t}=\max \left\{1, c \tau_{1}, c \tau_{2}\right\}, i=1,2$.

For any given $\lambda>0$, there exists a unique $\varepsilon_{i}=\varepsilon_{i}(\lambda)>0$ such that

$$
k_{i}-k_{i}\left(1-\varepsilon_{i}\right) e^{-\lambda t_{i}}=A_{i}, \quad i=1,2 .
$$

Then $\lim _{\lambda \rightarrow 0} k_{i} \varepsilon_{i}(\lambda)=A_{i}$ and

$$
e^{\lambda_{i} t}-q e^{\nu \lambda_{i} t} \geq k_{i}-k_{i}\left(1-\varepsilon_{i}\right) e^{-\lambda t}, \quad t_{i *} \leq t \leq t_{i}, \quad i=1,2 .
$$

We can see that for sufficiently small $\lambda$ and large enough $q>1$,

$$
0<\max \left\{\varepsilon_{1}, \varepsilon_{2}\right\} \ll \min \left\{1, \frac{1}{a_{2} k_{2}}, \frac{a_{1} k_{1}-b_{1} k_{2}}{a_{1} k_{1}}\right\} .
$$

Define the continuous functions as follows:

$$
\bar{\phi}(t)=\left\{\begin{array}{ll}
e^{\lambda_{1} t}, & t \leq t_{3}, \\
k_{1}+k_{1} e^{-\lambda t}, & t>t_{3},
\end{array} \quad \bar{\psi}(t)= \begin{cases}e^{\lambda_{2} t}+q e^{\nu \lambda_{2} t}, & t \leq t_{4}, \\
k_{2}+k_{2} e^{-\lambda t}, & t>t_{4},\end{cases}\right.
$$

and

$$
\underline{\phi}(t)=\left\{\begin{array}{ll}
e^{\lambda_{1} t}-q e^{\nu \lambda_{1} t}, & t \leq t_{1}, \\
k_{1}-k_{1}\left(1-\varepsilon_{1}\right) e^{-\lambda t}, & t>t_{1},
\end{array} \quad \underline{\psi}(t)= \begin{cases}e^{\lambda_{2} t}-q e^{\nu \lambda_{2} t}, & t \leq t_{2}, \\
k_{2}-k_{2}\left(1-\varepsilon_{2}\right) e^{-\lambda t}, & t>t_{2},\end{cases}\right.
$$

where $\lambda>0$ is small enough and $q>1$ is large enough, which will be determined later. Then

$$
M_{1}:=\sup _{t \in \mathbb{R}} \bar{\phi}(t)>k_{1}, \quad M_{2}:=\sup _{t \in \mathbb{R}} \bar{\psi}(t)>k_{2},
$$

$\bar{\phi}(t), \bar{\psi}(t), \underline{\phi}(t)$ and $\underline{\psi}(t)$ satisfy (A1) and (A2) and

$$
\max \left\{t_{1}+c \tau_{1}, t_{2}+c \tau_{2}\right\} \ll t_{4} \ll \min \left\{0, t_{3}\right\}
$$

for small enough $\lambda>0$ and large enough $q>1$. By the choice of $\nu$, we have $\Delta_{i}\left(\nu \lambda_{i}, c\right)<$ $0, i=1,2$.

Lemma 2.5. Assume that (2.6) holds. Then $(\bar{\phi}(t), \bar{\psi}(t))$ is a weak upper solution and $(\phi(t), \psi(t))$ is a weak lower solution of (2.1), respectively.

Proof. Without loss of generality, assume $\sigma_{k}>0$, otherwise, we only need to distinguish them from positive, negative or zero. Define

$$
\begin{aligned}
P(\phi, \psi)(t):= & D_{1} \sum_{k=1}^{n}\left[\phi\left(t+\sigma_{k}\right)-2 \phi(t)+\phi\left(t-\sigma_{k}\right)\right]-c \phi^{\prime}(t) \\
& +\alpha_{1} e^{-\gamma_{1} \tau_{1}} \phi\left(t-c \tau_{1}\right)-a_{1} \phi^{2}(t)-b_{1} \phi(t) \psi(t), \\
Q(\phi, \psi)(t):= & D_{2} \sum_{k=1}^{n}\left[\psi\left(t+\sigma_{k}\right)-2 \psi(t)+\psi\left(t-\sigma_{k}\right)\right]-c \psi^{\prime}(t) \\
& +\alpha_{2} e^{-\gamma_{2} \tau_{2}} \psi\left(t-c \tau_{2}\right)+b_{2} \phi(t) \psi(t)-a_{2} \psi^{2}(t) .
\end{aligned}
$$

We have two cases to verify for $\bar{\phi}(t)$.

(i) For $t<t_{3}$, since $\bar{\phi}\left(t \pm \sigma_{k}\right) \leq e^{\lambda_{1}\left(t \pm \sigma_{k}\right)}$ and $\bar{\phi}\left(t-c \tau_{1}\right) \leq e^{\lambda_{1}\left(t-c \tau_{1}\right)}$, it follows that

$$
\begin{aligned}
P(\bar{\phi}, \underline{\psi})(t) & \leq D_{1} \sum_{k=1}^{n}\left[\bar{\phi}\left(t+\sigma_{k}\right)-2 \bar{\phi}(t)+\bar{\phi}\left(t-\sigma_{k}\right)\right]-c \bar{\phi}^{\prime}(t)+\alpha_{1} e^{-\gamma_{1} \tau_{1}} \bar{\phi}\left(t-c \tau_{1}\right) \\
& \leq e^{\lambda_{1} t} \Delta_{1}\left(\lambda_{1}, c\right)=0 .
\end{aligned}
$$


(ii) For $t>t_{3}$, since $t_{3} \gg t_{2}+c \tau_{1}, \bar{\phi}\left(t \pm \sigma_{k}\right) \leq k_{1}+k_{1} e^{-\lambda\left(t \pm \sigma_{k}\right)}$ and $\bar{\phi}\left(t-c \tau_{1}\right) \leq$ $k_{1}+k_{1} e^{-\lambda\left(t-c \tau_{1}\right)}$, we have

$$
\begin{aligned}
P(\bar{\phi}, \underline{\psi})(t) \leq & e^{-\lambda t}\left\{k_{1}\left[D_{1} \sum_{k=1}^{n}\left(e^{\lambda \sigma_{k}}-2+e^{-\lambda \sigma_{k}}\right)+c \lambda\right]\right. \\
& \left.+k_{1}\left[\alpha_{1} e^{-\gamma_{1} \tau_{1}} e^{\lambda c \tau_{1}}-a_{1} k_{1}\left(2+e^{-\lambda t}\right)-b_{1} k_{2}\left(\varepsilon_{2}-\left(1-\varepsilon_{2}\right) e^{-\lambda t}\right)\right]\right\} \\
:= & e^{-\lambda t} I_{1}(\lambda) .
\end{aligned}
$$

$I_{1}(\lambda)<0$ for sufficiently small $\lambda$ since $I_{1}(0)=2 k_{1}\left(b_{1} k_{2}-a_{1} k_{1}-b_{1} k_{2} \varepsilon_{2}\right)<0$ by $(2.6)$.

For $\bar{\psi}(t)$, we also have two cases to verify.

(i) For $t<t_{4}$, because of $t_{4} \rightarrow-\infty$ as $q \rightarrow \infty$, we have

$$
\Pi_{1}(q):=\frac{1}{q} e^{\left(\frac{\lambda_{1}+\lambda_{2}}{\lambda_{1}}-\nu\right) \lambda_{1} t}+e^{\lambda_{1} t} \rightarrow 0 \text { as } q \rightarrow \infty .
$$

Since $\bar{\psi}\left(t \pm \sigma_{k}\right) \leq e^{\lambda_{2}\left(t \pm \sigma_{k}\right)}+q e^{\nu \lambda_{2}\left(t \pm \sigma_{k}\right)}, \bar{\psi}\left(t-c \tau_{2}\right) \leq e^{\lambda_{2}\left(t-c \tau_{2}\right)}+q e^{\nu \lambda_{2}\left(t-c \tau_{2}\right)}$ and $\bar{\phi}(t) \leq e^{\lambda_{1} t}$, it follows that for sufficiently large $q>1$,

$$
\begin{aligned}
Q(\bar{\phi}, \bar{\psi})(t) & \leq D_{2} \sum_{k=1}^{n}\left[\bar{\psi}\left(t+\sigma_{k}\right)-2 \bar{\psi}(t)+\bar{\psi}\left(t-\sigma_{k}\right)\right]-c \bar{\psi}^{\prime}(t)+\alpha_{2} e^{-\gamma_{2} \tau_{2}} \bar{\psi}\left(t-c \tau_{2}\right)+b_{2} \bar{\phi}(t) \bar{\psi}(t) \\
& \left.\leq q e^{\nu \lambda_{2} t}\left[\Delta_{2}\left(\nu \lambda_{2}, c\right)\right]+b_{2} \Pi_{1}(q)\right] \leq 0 .
\end{aligned}
$$

(ii) For $t>t_{4}$, since $\bar{\phi}(t) \leq k_{1}+k_{1} e^{-\lambda t}$ and $\bar{\psi}\left(t \pm \sigma_{k}\right) \leq k_{2}+k_{2} e^{-\lambda\left(t \pm \sigma_{k}\right)}, \bar{\psi}\left(t-c \tau_{2}\right) \leq$ $k_{2}+k_{2} e^{-\lambda\left(t-c \tau_{2}\right)}$, we have

$$
\begin{aligned}
Q(\bar{\phi}, \bar{\psi})(t) \leq & k_{2} e^{-\lambda t}\left[\sum_{k=1}^{n} D_{2}\left(e^{\lambda \sigma_{k}}-2+e^{-\lambda \sigma_{k}}\right)+c \lambda\right. \\
& \left.+\alpha_{2} e^{-\gamma_{2} \tau_{2}} e^{\lambda c \tau_{2}}+\left(b_{2} k_{1}-a_{2} k_{2}\right)\left(2+e^{-\lambda t}\right)\right] \\
:= & e^{-\lambda t} I_{2}(\lambda) .
\end{aligned}
$$

$I_{2}(\lambda)<0$ for sufficiently small $\lambda$ since $I_{2}(0)=2 k_{2}\left(b_{2} k_{1}-a_{2} k_{2}\right)=-2 k_{2} \alpha_{2} e^{-\gamma_{2} \tau_{2}}<0$.

We have two cases to verify for $\underline{\phi}(t)$.

(i) For $t<t_{1}<0$, because of $t_{1} \stackrel{-}{\rightarrow}-\infty$ as $q \rightarrow \infty$, we have

$$
\Pi_{2}(q):=\frac{a_{1}}{q} e^{(2-\nu) \lambda_{1} t}+b_{1}\left(\frac{1}{q} e^{\left(\frac{\lambda_{1}+\lambda_{2}}{\lambda_{1}}-\nu\right) \lambda_{1} t}+e^{\left(\lambda_{1}+\nu\left(\lambda_{2}-\lambda_{1}\right)\right) t}\right) \rightarrow 0 \text { as } q \rightarrow \infty .
$$

Since $\underline{\phi}\left(t \pm \sigma_{k}\right) \geq e^{\lambda_{1}\left(t \pm \sigma_{k}\right)}-q e^{\nu \lambda_{1}\left(t \pm \sigma_{k}\right)}, \bar{\phi}\left(t-c \tau_{1}\right) \geq e^{\lambda_{1}\left(t-c \tau_{1}\right)}-q e^{\nu \lambda_{1}\left(t-c \tau_{1}\right)}$ and $\bar{\psi}(t) \leq$ $e^{\lambda_{2} t}+q e^{\nu \lambda_{2} t}$, it follows that for sufficiently large $q>1$,

$$
\begin{aligned}
P(\underline{\phi}, \bar{\psi})(t) & \geq-q e^{\nu \lambda_{1} t} \Delta_{1}\left(\nu \lambda_{1}, c\right)+\left(e^{\lambda_{1} t}-q e^{\nu \lambda_{1} t}\right)\left[-a_{1}\left(e^{\lambda_{1} t}-q e^{\nu \lambda_{1} t}\right)-b_{1}\left(e^{\lambda_{2} t}+q e^{\nu \lambda_{2} t}\right)\right] \\
& \geq-q e^{\nu \lambda_{1} t}\left[\Delta_{1}\left(\nu \lambda_{1}, c\right)+r_{1} \Pi_{2}(q)\right] \geq 0 .
\end{aligned}
$$

(ii) For $t>t_{1}$, since $\bar{\psi}(t) \leq k_{2}+k_{2} e^{-\lambda t}$ and $\underline{\phi}\left(t \pm \sigma_{k}\right) \geq k_{1}-k_{1}\left(1-\varepsilon_{1}\right) e^{-\lambda\left(t \pm \sigma_{k}\right)}, \phi(t-$ $\left.c \tau_{1}\right) \geq k_{1}-k_{1}\left(1-\varepsilon_{1}\right) e^{-\lambda\left(t-c \tau_{1}\right)}$ by $(2.7)$ and $t_{1}-t_{1 *}>\tilde{t}$, we have

$$
\begin{aligned}
P(\underline{\phi}, \bar{\psi})(t) \geq & e^{-\lambda t}\left\{-k_{1}\left(1-\varepsilon_{1}\right)\left[D_{1} \sum_{k=1}^{n}\left(e^{\lambda \sigma_{k}}-2+e^{-\lambda \sigma_{k}}\right)+c \lambda\right]-k_{1}\left(1-\varepsilon_{1}\right) \alpha_{1} e^{-\gamma_{1} \tau_{1}} e^{\lambda c \tau_{1}}\right. \\
& \left.-a_{1} k_{1}^{2}\left[-2\left(1-\varepsilon_{1}\right)+\left(1-\varepsilon_{1}\right)^{2} e^{-\lambda t}\right]-b_{1} k_{1} k_{2}\left[\varepsilon_{1}-\left(1-\varepsilon_{1}\right) e^{-\lambda t}\right]\right\} \\
:= & e^{-\lambda t} I_{3}(\lambda) .
\end{aligned}
$$

$I_{3}(\lambda)>0$ for sufficiently small $\lambda$ since $I_{3}(0)=\varepsilon_{1} k_{1}\left(a_{1} k_{1}-b_{1} k_{2}-a_{1} k_{1} \varepsilon_{1}\right)>0$ by $(2.6)$ and $\varepsilon_{1}<\frac{a_{1} k_{1}-b_{1} k_{2}}{a_{1} k_{1}}$.

For $\underline{\psi}(t)$, we only have two cases to verify. 
(i) For $t<t_{2}<0$, since $\psi\left(t \pm \sigma_{k}\right) \geq e^{\lambda_{2}\left(t \pm \sigma_{k}\right)}-q e^{\nu \lambda_{2}\left(t \pm \sigma_{k}\right)}, \psi\left(t-c \tau_{2}\right) \geq e^{\lambda_{2}\left(t-c \tau_{2}\right)}-$ $q e^{\nu \lambda_{2}\left(t-c \tau_{2}\right)}$ and $\psi(t) \leq e^{\lambda_{2} t}$, for sufficiently large $q>1$, we have

$$
\begin{aligned}
Q(\underline{\phi}, \underline{\psi})(t) & \geq D_{2} \sum_{k=1}^{n}\left[\underline{\psi}\left(t+\sigma_{k}\right)-\underline{\psi}(t)+\underline{\psi}\left(t-\sigma_{k}\right)\right]-c \underline{\psi}(t)+\alpha_{2} e^{-\gamma_{2} \tau_{2}} \underline{\psi}\left(t-c \tau_{2}\right)-a_{2} \underline{\psi}^{2}(t) \\
& \geq-q e^{\nu \lambda_{2} t}\left[\Delta_{2}\left(\nu \lambda_{2}, c\right)+\frac{a_{2}}{q} e^{(2-\nu) \lambda_{2} t}\right] \geq 0 .
\end{aligned}
$$

(ii) For $t>t_{2}$, we can divide this case into two subcases: (a) $t_{2}<t \leq \max \left\{t_{1}, t_{2}\right\}$, (b) $t>\max \left\{t_{1}, t_{2}\right\}$. In fact, if $t_{2} \geq t_{1}$, it only has case (b).

(a) In view of $t_{4} \rightarrow 0$ as $q \rightarrow \infty, \underline{\psi}\left(t \pm \sigma_{k}\right) \geq k_{2}-k_{2}\left(1-\varepsilon_{2}\right) e^{-\lambda\left(t \pm \sigma_{k}\right)}, \underline{\psi}\left(t-c \tau_{2}\right) \geq$ $k_{2}-k_{2}\left(1-\varepsilon_{2}\right) e^{-\lambda\left(t-c \tau_{2}\right)}$ by $(2.7)$ and $\bar{t}_{2}-t_{2 *}>\tilde{t}$, it follows that

$$
\begin{aligned}
Q(\underline{\phi}, \underline{\psi})(t) \geq & -k_{2}\left(1-\varepsilon_{2}\right)\left[D_{2} \sum_{k=1}^{n}\left(e^{\lambda \sigma_{k}}-2+e^{-\lambda \sigma_{k}}\right)+c \lambda\right] e^{-\lambda t} \\
& +\alpha_{2} e^{-\gamma_{2} \tau_{2}}\left[k_{2}-k_{2}\left(1-\varepsilon_{2}\right) e^{-\lambda\left(t-c \tau_{2}\right)}\right]-a_{2}\left[k_{2}-k_{2}\left(1-\varepsilon_{2}\right) e^{-\lambda t}\right]^{2} \\
& \rightarrow \alpha_{2} e^{-\gamma_{2} \tau_{2}} A_{2}-a_{2} A_{2}^{2} \text { as } \lambda \rightarrow 0 .
\end{aligned}
$$

Then $Q(\phi, \psi)(t) \geq 0$ for sufficiently small $\lambda>0$ since $\lambda$ is independent of $q$ and $A_{2} \rightarrow 0$ as $q \rightarrow \infty$.

(b) we can get

$$
\begin{aligned}
Q(\underline{\phi}, \underline{\psi})(t) \geq & e^{-\lambda t}\left\{-k_{2}\left(1-\varepsilon_{2}\right)\left[D_{2} \sum_{k=1}^{n}\left(e^{\lambda \sigma_{k}}-2+e^{-\lambda \sigma_{k}}\right)+c \lambda\right]\right. \\
& -k_{2}\left(1-\varepsilon_{2}\right) \alpha_{2} e^{-\gamma_{2} \tau_{2}} e^{\lambda c \tau_{2}}+b_{1} k_{1} k_{2}\left[-\left(1-\varepsilon_{1}\right)-\left(1-\varepsilon_{2}\right)+\left(1-\varepsilon_{1}\right)\left(1-\varepsilon_{2}\right) e^{-\lambda t}\right] \\
& \left.-a_{2} k_{2}^{2}\left[-2\left(1-\varepsilon_{2}\right)+\left(1-\varepsilon_{2}\right)^{2} e^{-\lambda t}\right]\right\} \\
:= & e^{-\lambda t} I_{4}(\lambda) .
\end{aligned}
$$

$I_{4}(\lambda)>0$ for sufficiently small $\lambda$ since $I_{4}(0)=\varepsilon_{2} k_{2}\left(1-a_{2} k_{2} \varepsilon_{2}+b_{2} k_{1} \varepsilon_{1}\right)>0$ by $a_{2} k_{2}-b_{2} k_{1}=$ $\alpha_{2} e^{-\gamma_{2} \tau_{2}}$ and $\varepsilon_{2}<\frac{1}{a_{2} k_{2}}$.

Define

$\Gamma([\underline{\phi}, \underline{\psi}],[\bar{\phi}, \bar{\psi}])=\left\{(\phi, \psi) \in C_{[\mathbf{0}, \mathbf{M}]}\left(\mathbb{R}, \mathbb{R}^{2}\right) \mid(\underline{\phi}(t), \underline{\psi}(t)) \leq(\phi(t), \psi(t)) \leq(\bar{\phi}(t), \bar{\psi}(t)), \quad t \in \mathbb{R}\right\}$.

Obviously, $\Gamma([\phi, \psi],[\bar{\phi}, \bar{\psi}])$ is nonempty.

Similar to the proofs in $[8,9,11,12]$, we have the following lemma.

Lemma 2.6. $F$ has the following properties:

(i) $F_{1}\left(\phi_{2}, \psi_{1}\right)(t) \leq F_{1}\left(\phi_{1}, \psi_{2}\right)(t), \quad F_{2}\left(\phi_{1}, \psi_{2}\right)(t) \leq F_{2}\left(\phi_{2}, \psi_{1}\right)(t)$ for $t \in \mathbb{R}$ if $\left(\phi_{i}, \psi_{i}\right) \in C_{[0, M]}\left(\mathbb{R}, \mathbb{R}^{2}\right)$ satisfy $\boldsymbol{O} \leq\left(\phi_{2}(t), \psi_{2}(t)\right) \leq\left(\phi_{1}(t), \psi_{1}(t)\right) \leq \boldsymbol{M}$ for $t \in \mathbb{R}, i=1,2$;

(ii) $F=\left(F_{1}, F_{2}\right): C_{[0, M]}\left(\mathbb{R}, \mathbb{R}^{2}\right) \rightarrow C\left(\mathbb{R}, \mathbb{R}^{2}\right)$ is continuous with respect to the norm $|\cdot|_{\mu}$ in $B_{\mu}\left(\mathbb{R}, \mathbb{R}^{2}\right)$;

(iii) $F(\Gamma([\underline{\phi}, \underline{\psi}],[\bar{\phi}, \bar{\psi}])) \subset \Gamma([\phi, \underline{\psi}],[\bar{\phi}, \bar{\psi}])$;

(iv) $F: \Gamma([\underline{\phi}, \underline{\psi}],[\bar{\phi}, \bar{\psi}]) \rightarrow \Gamma([\underline{\phi}, \underline{\psi}],[\bar{\phi}, \bar{\psi}])$ is compact with respect to the norm $|\cdot|_{\mu}$.

By Schauder's fixed point theorem, we have the following existence result.

Theorem 2.7. Assume that (2.6) holds. Then, for every $c>c^{*}$, (1.1) has a traveling wave solution $(\phi(\sigma \cdot \eta+c t), \psi(\sigma \cdot \eta+c t))$ with the wave speed $c$ which connects $\boldsymbol{O}$ with $\boldsymbol{K}$. Moreover, $\lim _{\xi \rightarrow-\infty}\left(\phi(\xi) e^{-\lambda_{1} \xi}, \psi(\xi) e^{-\lambda_{2} \xi}\right)=(1,1)$, where $\xi=\sigma \cdot \eta+$ ct. 
Acknowledgment. We would like to thank the anonymous referees for their careful reading and helpful suggestions which led to an improvement of the original manuscript. This research was supported by the National Natural Science Foundation of China (Grant No. 11971160), the Scientific Research Fund of Hunan Provincial Education Department (Grant No. 18B472) and the Scientific Research Fund of Hunan First Normal University (Grant No. XYS14N08).

\section{References}

[1] J. Al-Omari and S.A. Gourly, Monotone travelling fronts in an age-structured reaction-diffusion model of a single species, J. Math. Biol. 45 (2), 294-312, 2002.

[2] J. Al-Omari and S.A. Gourly, Stability and traveling fronts in Lotka-Volterra competition models with stage structure, SIAM J. Appl. Math. 63 (6), 2063-2086, 2003.

[3] C.P. Cheng, W.T. Li, Z.C. Wang and S.Z. Zheng, Traveling waves connecting equilibrium and periodic orbit for a delayed population model on a two-dimensional spatial lattice, Int. J. Bifurcat. Chaos Appl. Sci. Engrg. 26 (3), 1650049, 2016.

[4] S.N. Chow, J. Mallet-Paret and W. Shen, Traveling waves in lattice dynamical systems, J. Differential Equations 149 (2), 248-291, 1998.

[5] S.A. Gourley and Y. Kuang, Wavefronts and global stability in a time-delayed population model with stage structure, Proc. R. Soc. Lond. Ser. A 459, 1563-1579, 2003.

[6] S.A. Gourley and Y. Kuang, A stage structured predator-prey model and its dependence on maturation delay and death rate, J. Math. Biol. 49 (2), 188-200, 2004.

[7] J. Guo and C. Wu, Traveling wave front for a two-component lattice dynamical system arising in competition models, J. Differential Equations 252 (8), 4357-4391, 2012.

[8] J. Huang, G. Lu and S. Ruan, Traveling wave solutions in delayed lattice differential equations with partial monotonicity, Nonlinear Anal. TMA 60 (7), 1331-1350, 2005.

[9] J. Huang and X. Zou, Travelling wave solutions in delayed reaction diffusion systems with partial monotonicity, Acta Math. Appl. Sin. Engl. Ser. 22 (2), 243-256, 2006.

[10] Y. Kuang and J, So, Analysis of a delayed two-stage population with space-limited recruitment, SIAM J. Appl. Math. 55 (6), 1675-1695, 1995.

[11] K. Li and X. Li, Traveling wave solutions in a reaction-diffusion competitioncooperation system with stage structure, Jpn J. Ind. Appl. Math. 35 (1), 157-193, 2018.

[12] K. Li and X. Li, Traveling wave solutions in a delayed lattice competition-cooperation system, J. Difference Equ. Appl. 24 (3), 391-408, 2018.

[13] G. Lin and W.T. Li, Traveling waves in delayed lattice dynamical systems with competition interactions, Nonlinear Anal. RWA 11 (5), 3666-3679, 2010.

[14] J.W.H. So, J. Wu and X. Zou, A reaction-diffusion model for a single species with age structure. I, Travelling wavefronts on unbounded domains, Proc. R. Soc. Lond. Ser. A 457 (2012), 1841-1853, 2001.

[15] S.L. Wu and S.Y. Liu, Travelling waves in delayed reaction-diffusion equations on higher dimensional lattices, J. Difference Equ. Appl. 19 (3), 384-401, 2013.

[16] J. Wu and X. Zou, Asymptotic and periodic boundary value problems of mixed FDEs and wave solutions of lattice differential equations, J. Differential Equations 135 (2), 315-357, 1997.

[17] Z.X. Yu and R. Yuan, Nonlinear stability of wavefronts for a delayed stage-structured population model on a 2-D lattice, Osaka J. Math. 50 (4), 963-976, 2013.

[18] L. Zhang and B.T. Li, Traveling wave solutions in an integro-differential competition model, Discrete Contin. Dyn. Syst. Ser. B 17 (1), 417-428, 2012.

[19] L. Zhang and B.T. Li, J. Shang, Stability and travelling wave solutions for a timedelayed population system with stage structure, Nonlinear Anal. RWA 13 (3), 14291440, 2012. 
[20] H.Q. Zhao, Asymptotic stability of traveling fronts in delayed reaction-diffusion monostable equations on higher-dimensional lattices, Electron. J. Differential Equations, 2013 (119), 1-15, 2013.

[21] H.Q. Zhao and S.L. Wu, Wave propagation for a reaction-diffusion model with a quiescent stage on a 2D spatial lattice, Nonlinear Anal. RWA 12 (2), 1178-1191, 2011.

[22] X. Zou, Traveling wave fronts in spatially discrete reaction-diffusion equations on higher-dimensional lattices, Proceedings of the Third Mississippi State Conference on Difference Equations and Computational Simulations, Mississippi State, Electron. J. Differ. Equ. Conf. 1, 211-221, 1997. 